\title{
Method of order reduction in second order differential equations line with GeoGebra
}

\author{
Jorge Olivares Funes ${ }^{1, *}$, Elvis Ronald Valero Kari ${ }^{2}$ \\ ${ }^{1}$ Universidad de Antofagasta, Antofagasta-Chile \\ ${ }^{2}$ Elvis Ronlad Valero Kari, Arica-Chile
}

\begin{abstract}
.
Various authors have released the wonderful animated applets that can be built from Several authors have shown the wonderful animated applets that can be built from certain content for teaching and learning, as for example Tom Walsh (1), Dan MacIsaac (2).

These authors in their work tell us how friendly it is to have GeoGebra applets in favoring the understanding of the content that one intends to teach.

In this papers, the GeoGebra applets will show how the second algebraic and graphical solution of a second order linear equation is, when order reduction is applied, which already has a known solution.

Reducing the order itself is of great help in ODEs, since in some cases it is not so easy to calculate the solutions of the homogeneous part of an ED, so the GeoGebra software gives us all its potential and dynamism to to be able to visualize these solutions through their applets and instruction commands.
\end{abstract}

All these applets were built as teaching support material for the differential equations course of engineering careers at the University of Antofagasta, during the year 2020.

Keywords: applets; differential equation; GeoGebra; order reduction; teaching.

\section{Introduction}

As we mentioned at the beginning the authors Tom Walsh (1), Dan MacIsaac (2).

They show us the advantages of using GeoGebra, especially Tom Wash (1) who tells us

that GeoGebra allows us to have "dynamic mathematics for teaching and learning " Thus, we will visualize the solutions obtained by using order reduction to second order

linear differential equations using GeoGebra applets.

This paper is a continuation and extension of animations and interactive creations in first order linear differential equations: the case of GeoGebra (3)

\section{GeoGebra and Order reduction in differential equations}

Below are several examples of the solutions obtained by order reduction and by GeoGebra applets, available at https://www.geogebra.org/m/w5vsmxrk 


\section{Example 1}

$\mathrm{Be}$

$$
\frac{d^{2} y}{d x^{2}}-\mathrm{y}=0, y_{1}=e^{x}
$$

In figure 1 The second solution obtained by reduction of order $\mathrm{e}^{\wedge}-\mathrm{x}$ is given in red and the general solution in blue. Where c_1 and c_2 move between -5 and 5 .

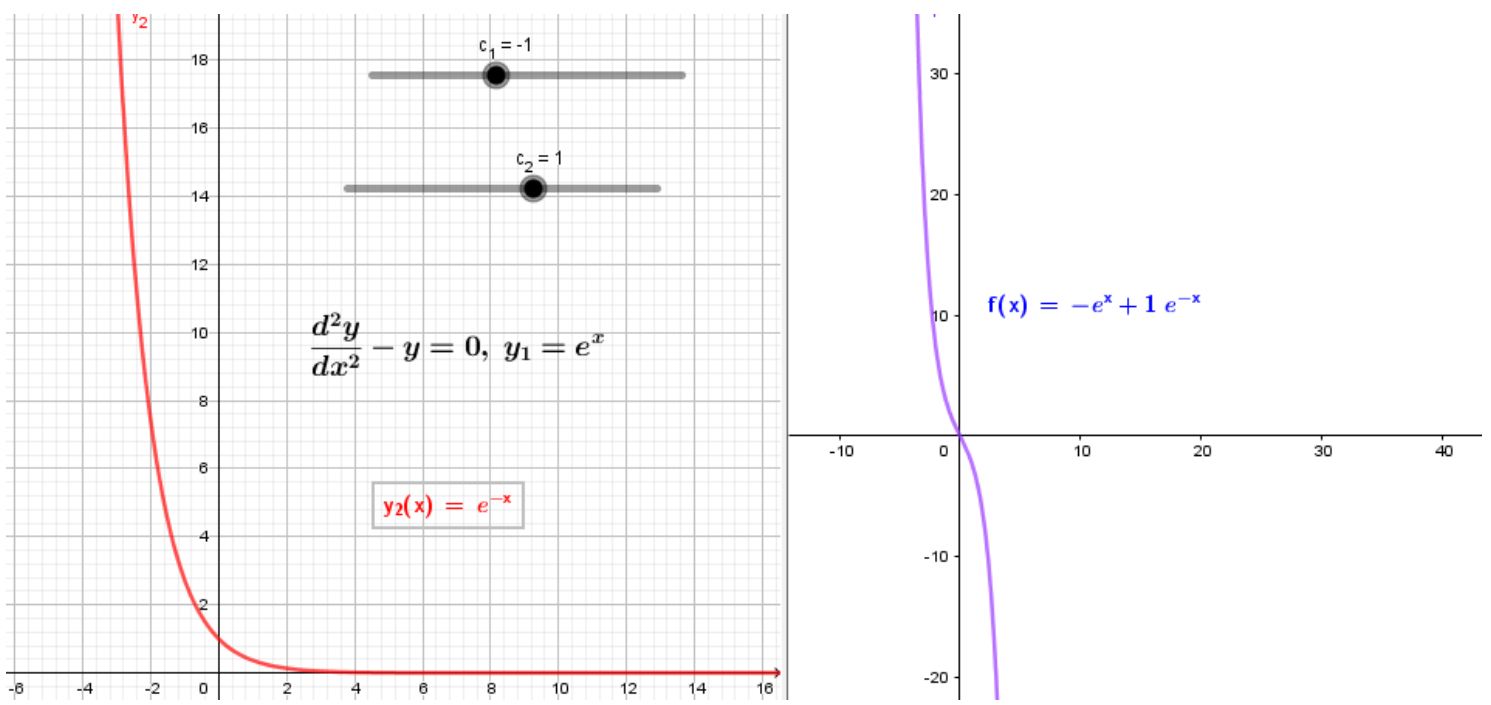

Figure 1

\section{Example 2}

$\mathrm{Be}$

$$
\frac{d^{2} y}{d x^{2}}-9 \mathrm{y}=0, y_{1}=e^{3 x}
$$

In figure 2 the second solution obtained by reduction of order $\mathrm{e}^{\wedge}-3 \mathrm{x}$ is given in red and the general solution in blue. Where c_1 and c_2 move between -5 and 5 . 


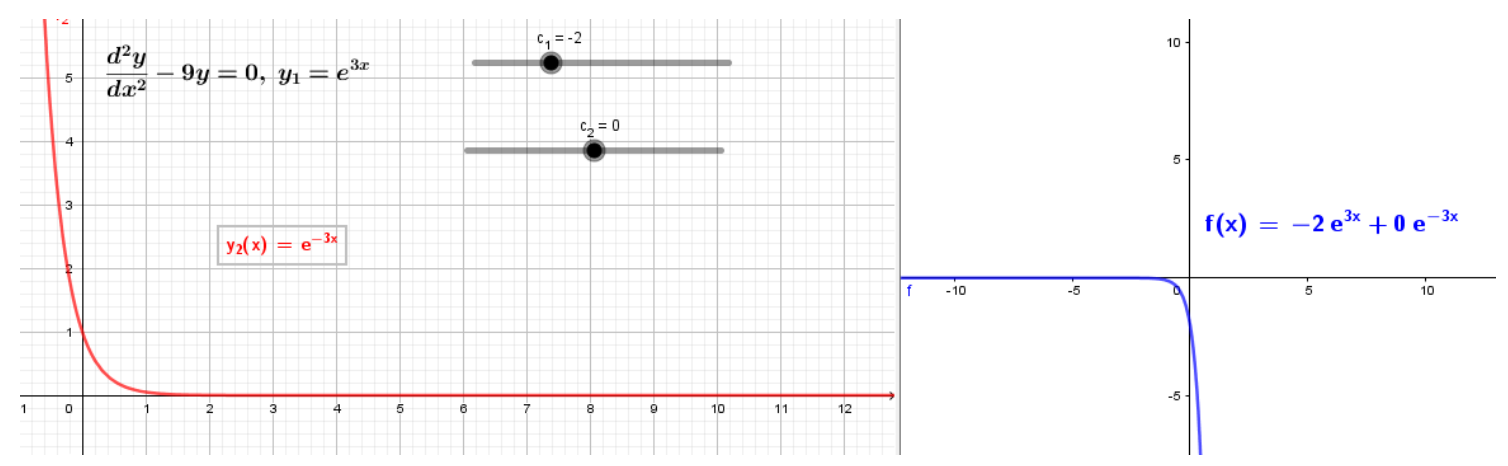

Figure 2

\section{Example 3}

$\mathrm{Be}$

$$
\frac{d^{2} y}{d x^{2}}-16 \mathrm{y}=0, y_{1}=e^{4 x}
$$

In figure 3 the second solution obtained by reduction of order $\mathrm{e}^{\wedge}-4 \mathrm{x}$ is given in red and the general solution in blue. Where c_1 and c_2 move between -5 and 5 .

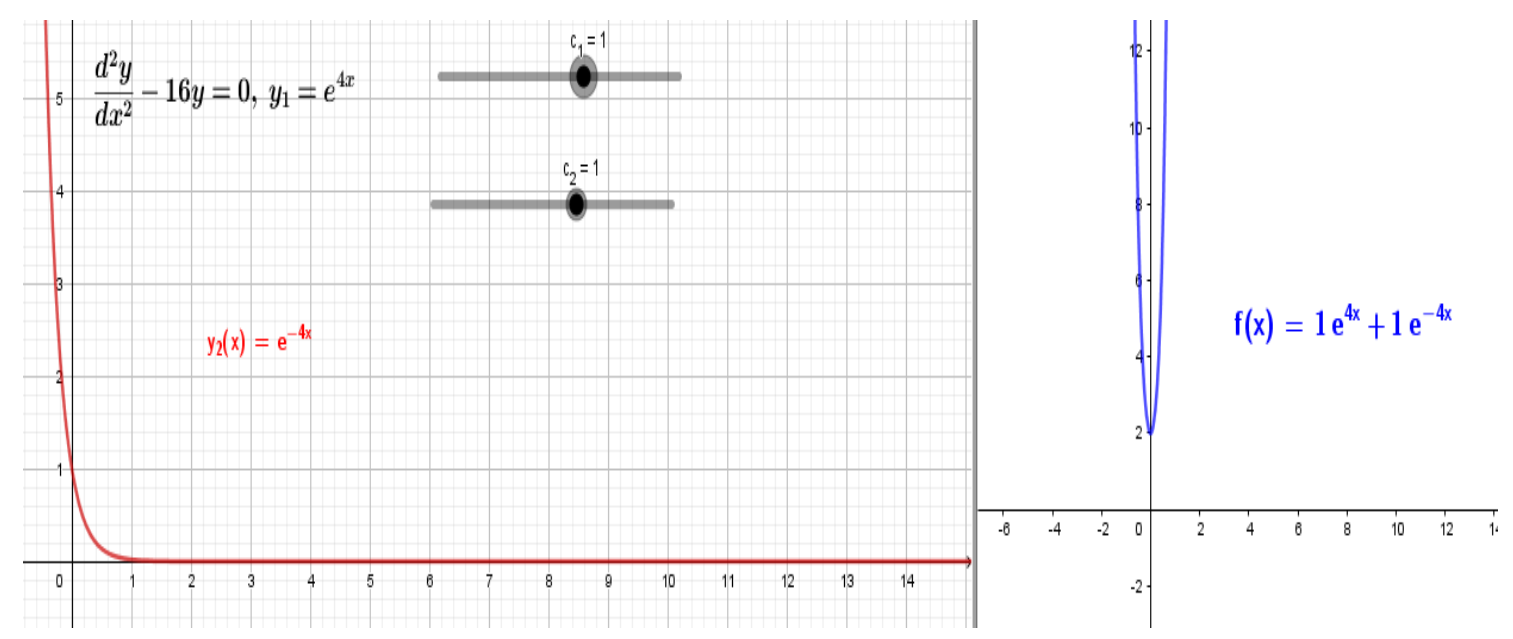

Figure 3

\section{Example 4}

$\mathrm{Be}$

$$
\frac{d^{2} y}{d x^{2}}-25 y=0, y_{1}=e^{5 x}
$$


In figure 4 the second solution obtained by reduction of order $e^{\wedge}\{-5 x\}$ is given in red and the general solution in blue. Where c_1 and c_2 move between -5 and 5 .

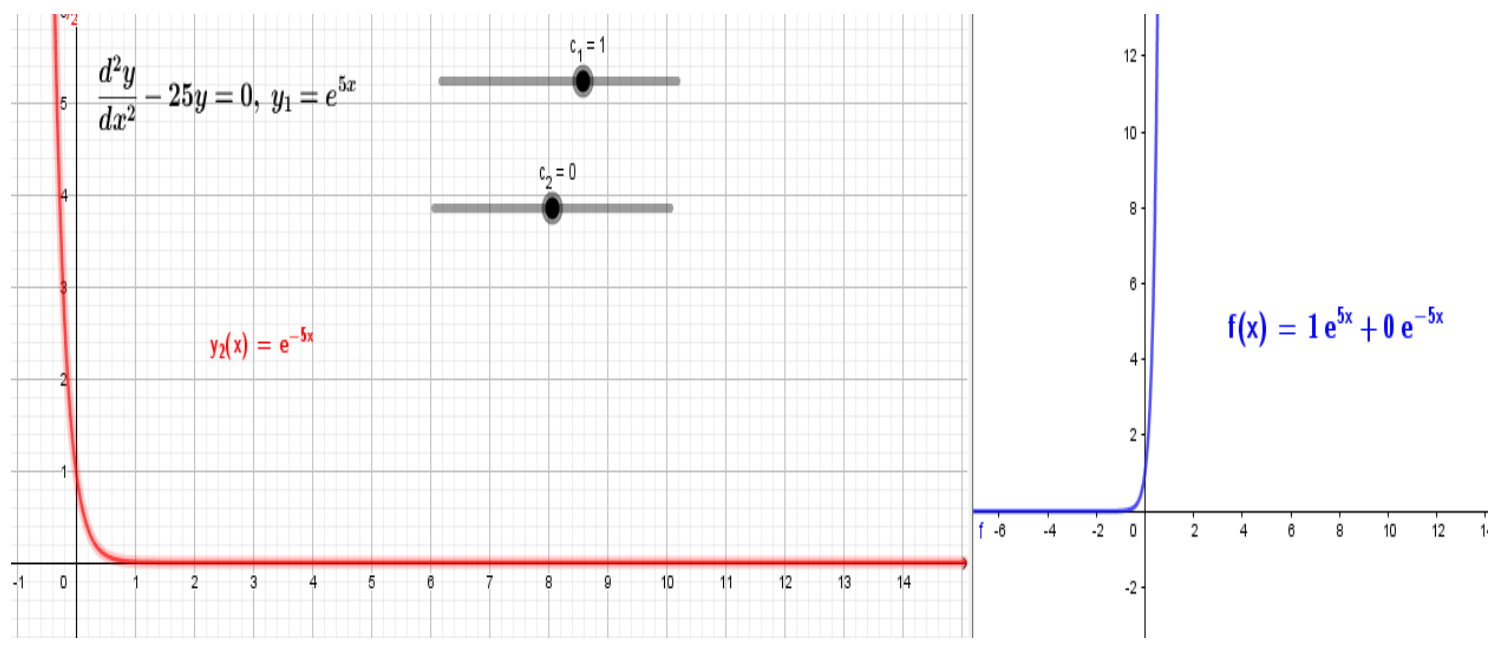

Figure 4

\section{Example 5}

$\mathrm{Be}$

$$
\frac{d^{2} y}{d x^{2}}-36 y=0, y_{1}=e^{6 x}
$$

In figure 5 the second solution obtained by reduction of order $\mathrm{e}^{\wedge}-6 \mathrm{x}$ is given in red and the general solution in blue. Where c_1 and c_2 move between -5 and 5 .

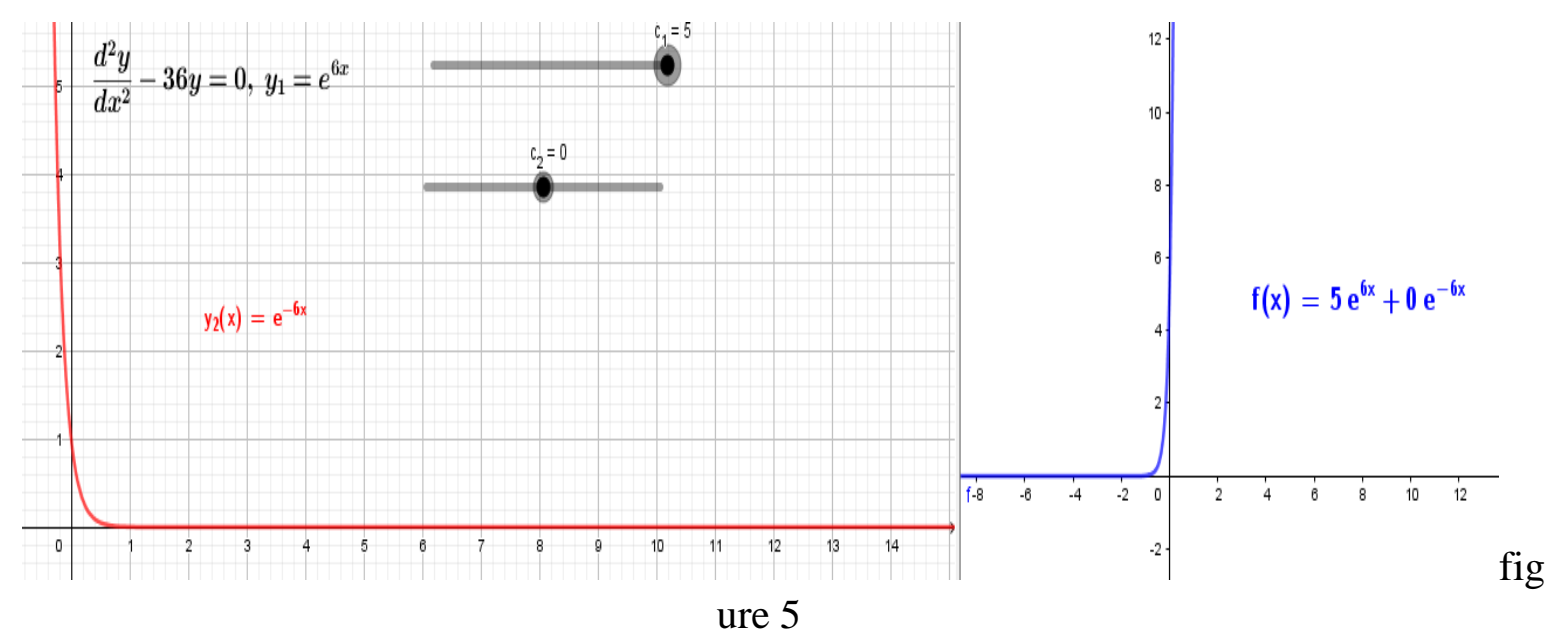




\section{Example 6}

$\mathrm{Be}$

$$
\frac{d^{2} y}{d x^{2}}+169 \mathrm{y}=0, y_{1}=\operatorname{sen} 13 x
$$

In figure $6 \mathrm{t}$ he second solution obtained by reduction of order $\cos 13 \mathrm{x}$ is given in red and the general solution in blue. Where c_1 and c_2 move between -5 and 5 .

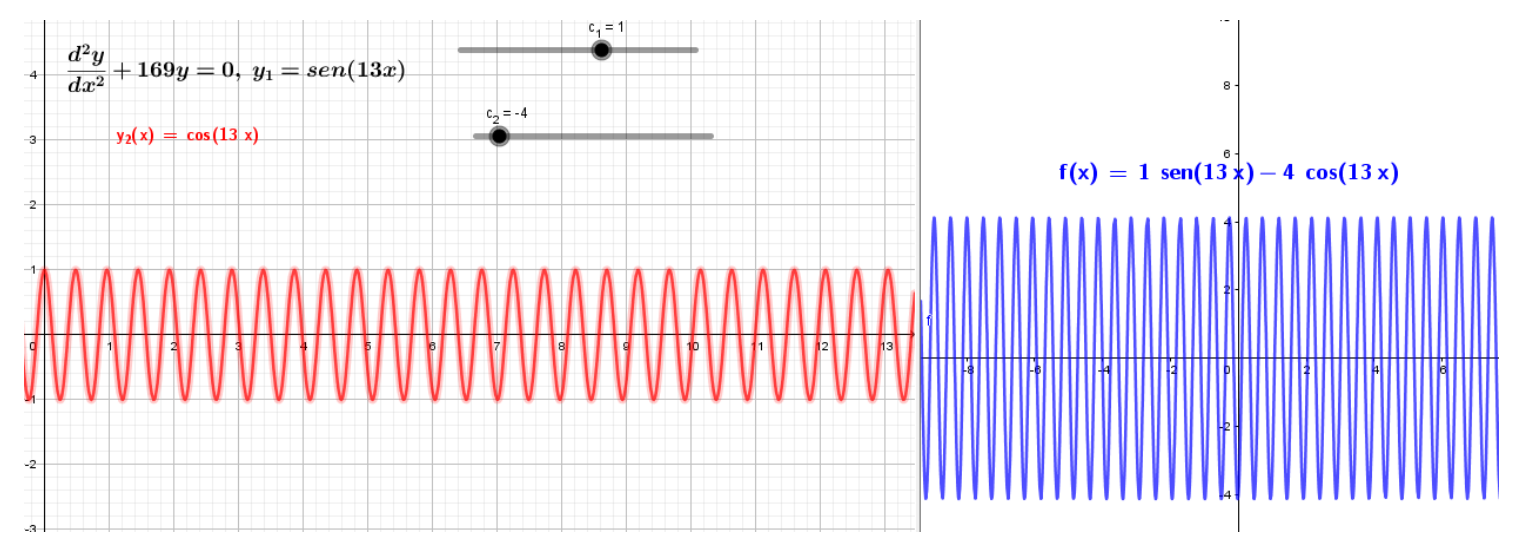

Figure 6

\section{Example 7}

$\mathrm{Be}$

$$
\frac{d^{2} y}{d x^{2}}+9 y=0, y_{1}=\operatorname{sen} 3 x
$$

In figure 7 the second solution obtained by reduction of order $\cos 3 x$ is given in red and the general solution in blue. Where c_1 and c_2 move between -5 and 5 . 


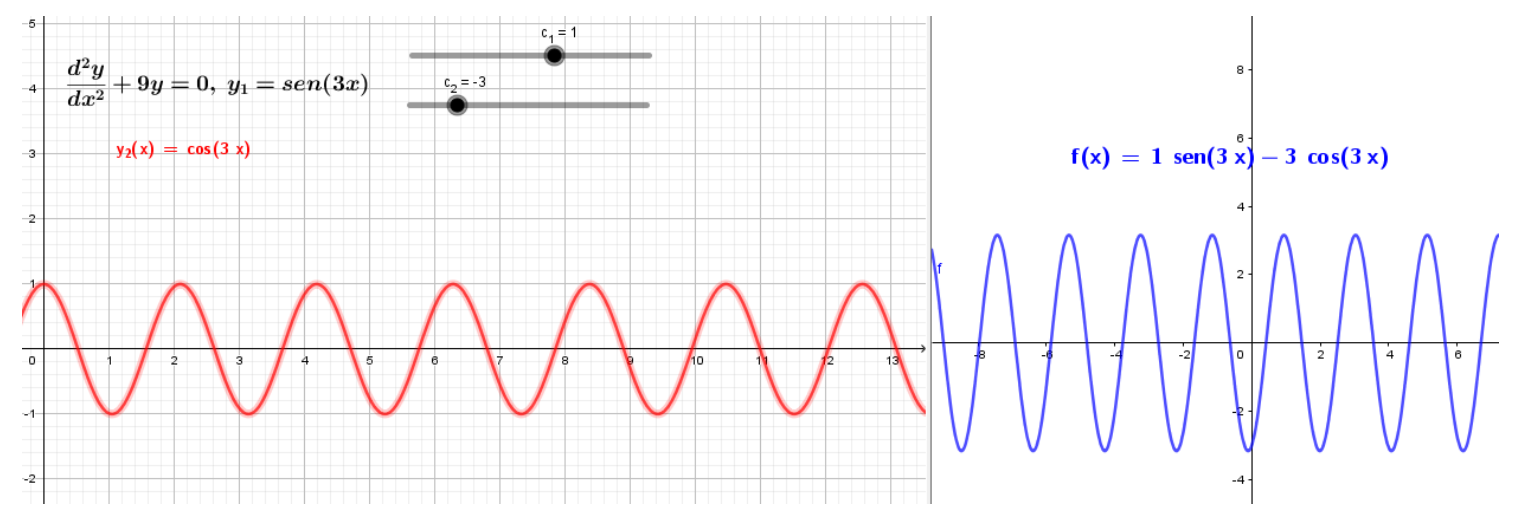

Figure 7

\section{Example 8}

$\mathrm{Be}$

$$
\frac{d^{2} y}{d x^{2}}+100 \mathrm{y}=0, y_{1}=\operatorname{sen} 10 x
$$

In figure 8 the second solution obtained by reduction of order $\cos 10 \mathrm{x}$ is given in red and the general solution in blue. Where c_1 and c_2 move between -5 and 5 .

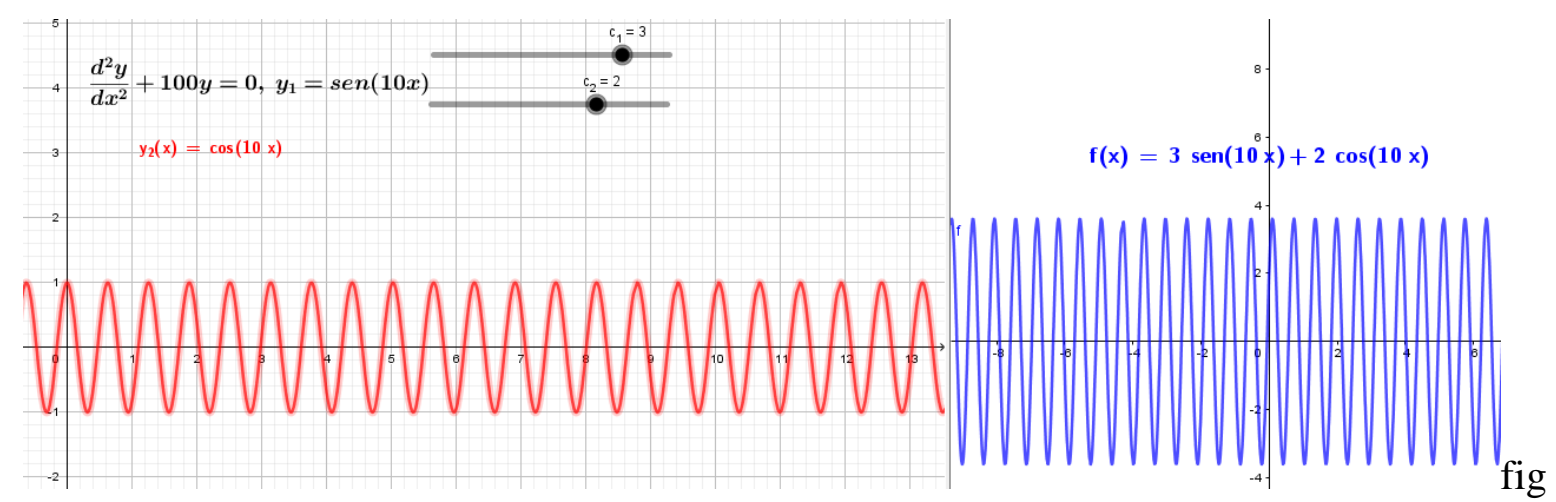

Figure 8

\section{Example 9}

$\mathrm{Be}$

$$
\frac{d^{2} y}{d x^{2}}+\mathrm{y}=0, y_{1}=\operatorname{sen} x
$$

In figure 9 the second solution obtained by reduction of order cosx is given in red and the general solution in blue. Where c_1 and c_2 move between -5 and 5 . 


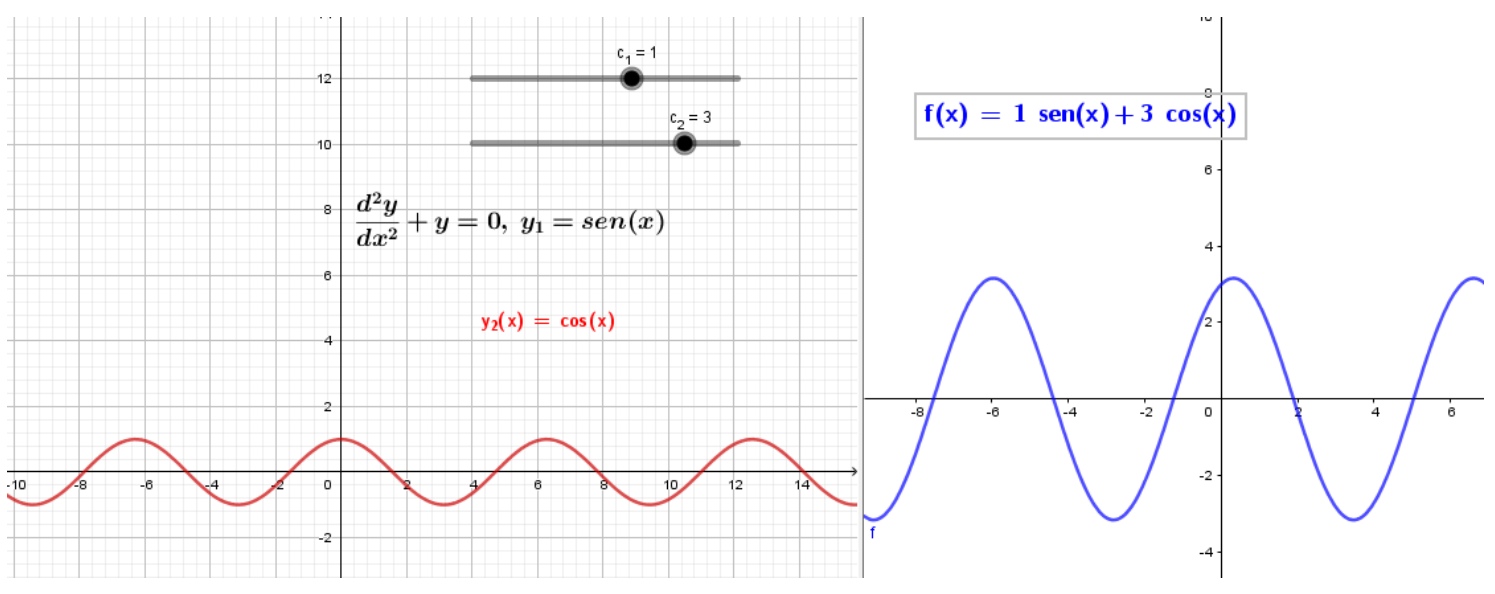

Figure 9

\section{Example 10}

$\mathrm{Be}$

$$
\frac{d^{2} y}{d x^{2}}+\mathrm{y} / 4=0, y_{1}=\operatorname{sen}(x / 2)
$$

In figure 10 the second solution obtained by reduction of order $\cos (\mathrm{x} / 2)$ is given in red and the general solution in blue. Where c_1 and c_2 move between -5 and 5 .

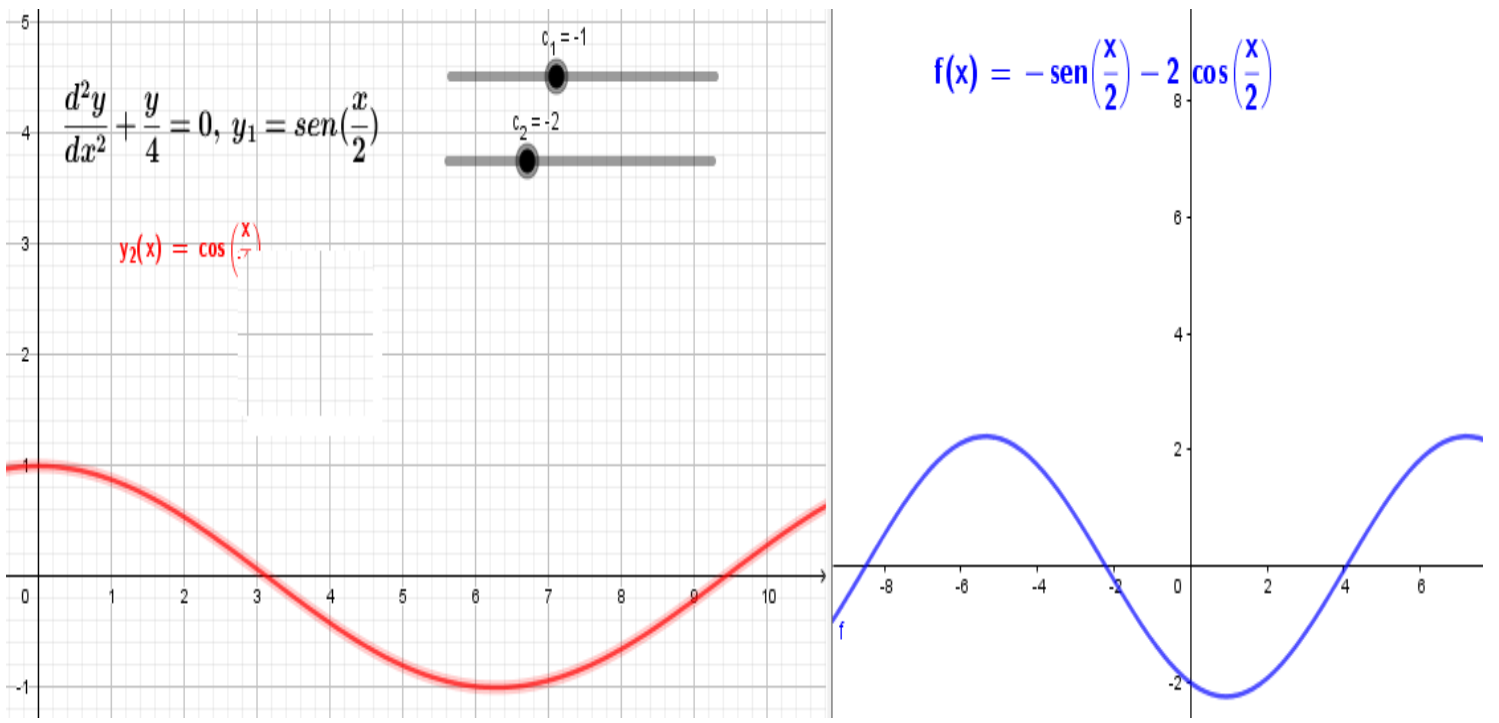

figure 10 


\section{Example 11}

$\mathrm{Be}$

$$
\frac{d^{2} y}{d x^{2}}+\mathrm{y} / 16=0, y_{1}=\operatorname{sen}(x / 4)
$$

In figure 11 the second solution obtained by reduction of order $\cos (\mathrm{x} / 4)$ is given in red and the general solution in blue. Where c_1 and c_2 move between -5 and 5 .

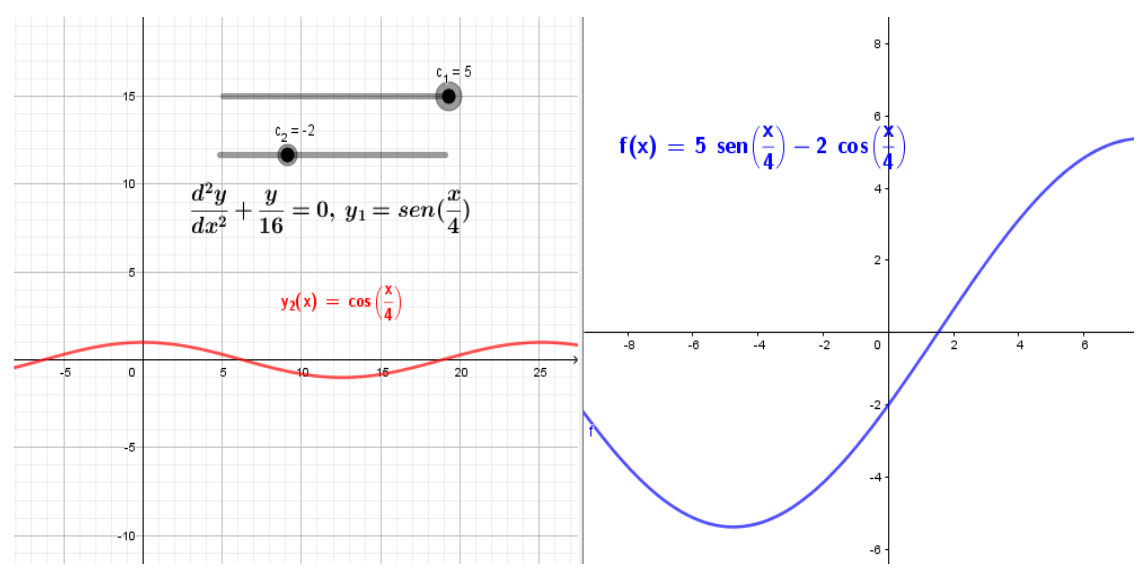

Figure 11

\section{Example 12}

$\mathrm{Be}$

$$
\frac{d^{2} y}{d x^{2}}+\mathrm{y} / 81=0, y_{1}=\operatorname{sen}(x / 9)
$$

In figure 12 the second solution obtained by reduction of order $\cos (x / 9)$ is given in red and the general solution in blue. Where c_1 and c_2 move between -5 and 5 . 


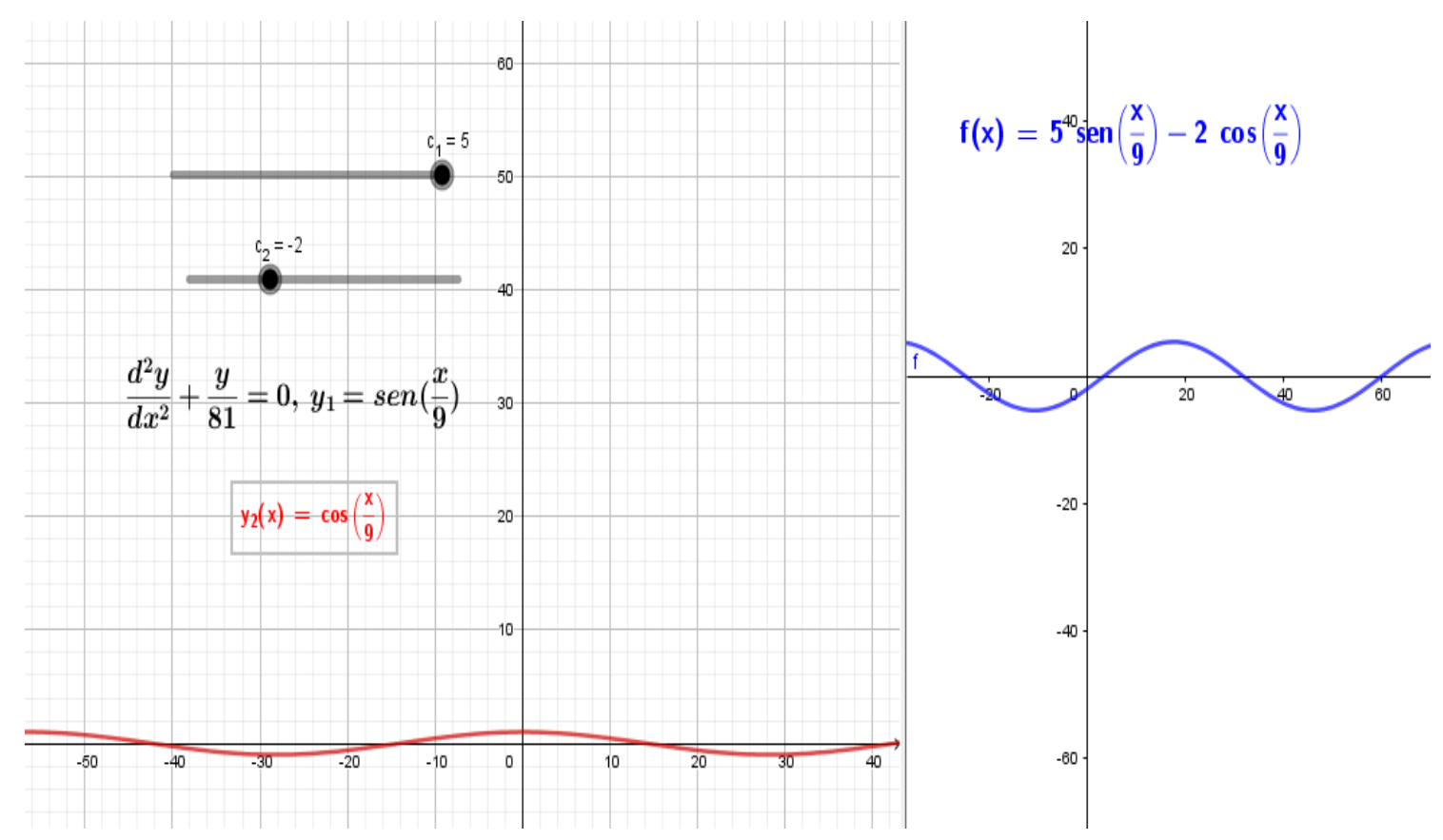

Figure 12

\section{Example 13}

$\mathrm{Be}$

$$
\frac{d^{2} y}{d x^{2}}+\mathrm{y} / 49=0, y_{1}=\operatorname{sen}(x / 7)
$$

In figure 13 the second solution obtained by reduction of order $\cos (x / 7)$ is given in red and the general solution in blue. Where c_1 and c_2 move between -5 and 5 . 


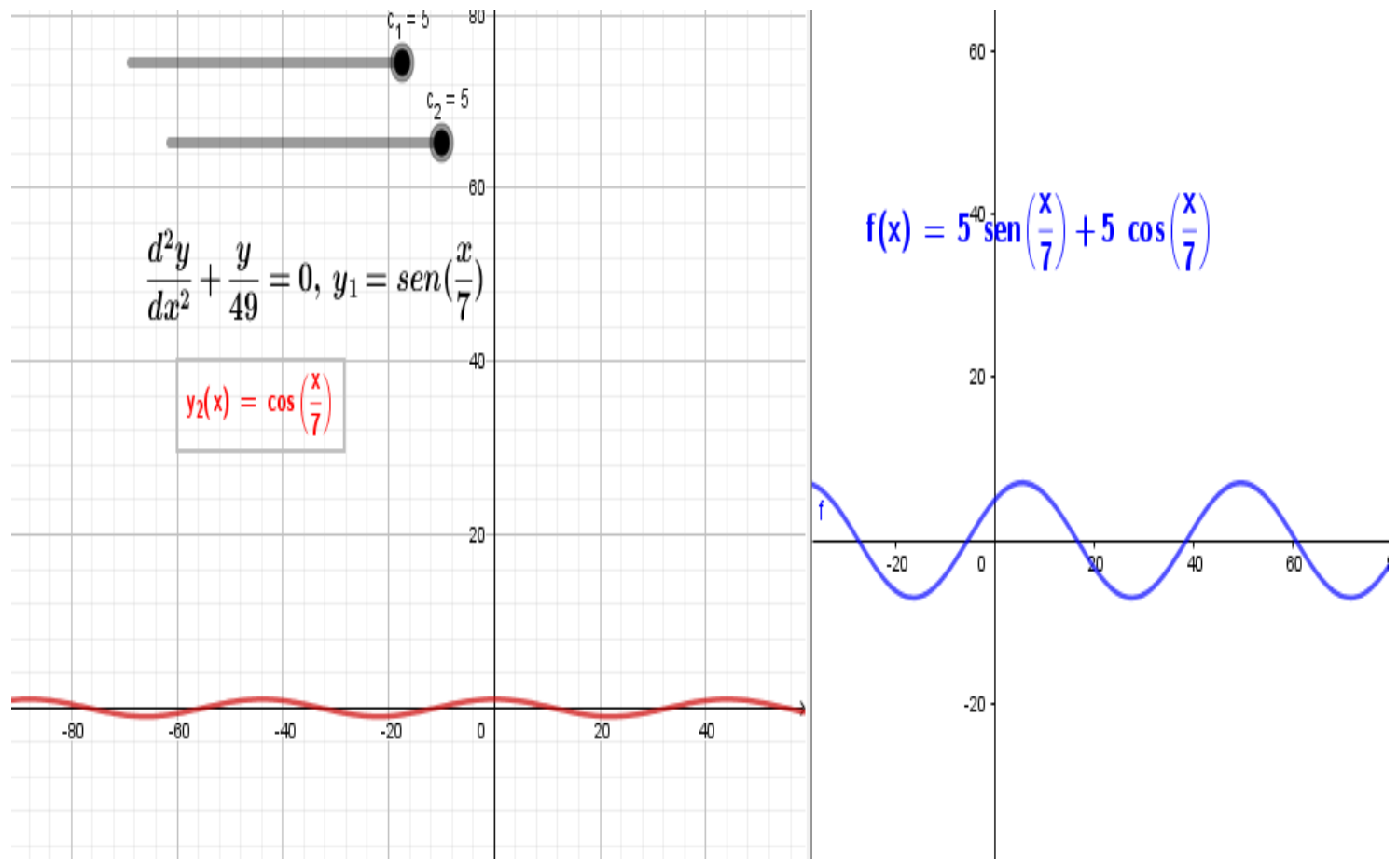

Figure 13

\section{Example 14}

$\mathrm{Be}$

$$
x \frac{d^{2} y}{d x^{2}}+\frac{d y}{d x}=0, y_{1}=\ln x
$$

By order reduction, the second solution is $y_{2}=-1$ which is given in red in Figure 14 .

Where the delinkers ${ }^{\mathrm{I}} c_{1}$ y $c_{2}{ }^{\mathrm{I}}$ move between -5 and 5 in the general solution $y_{s}$.
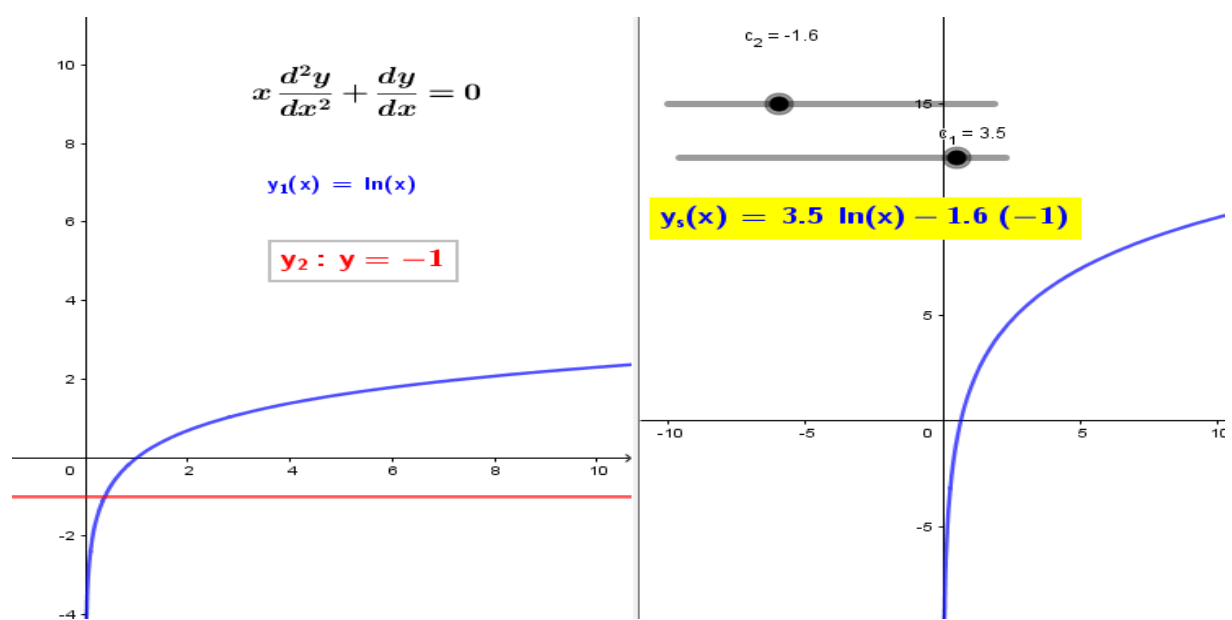

www.worldte.org info@worldte.org 
Figure 14

\section{Example 15}

$\mathrm{Be}$

$$
\frac{d^{2} y}{d x^{2}}+\mathrm{y} / 256=0, y_{1}=\operatorname{sen}(x / 16)
$$

In figure 15 the second solution obtained by reduction of order $\cos (\mathrm{x} / 16)$ is given in red and the general solution in blue. Where c_1 and c_2 move between -5 and 5 .

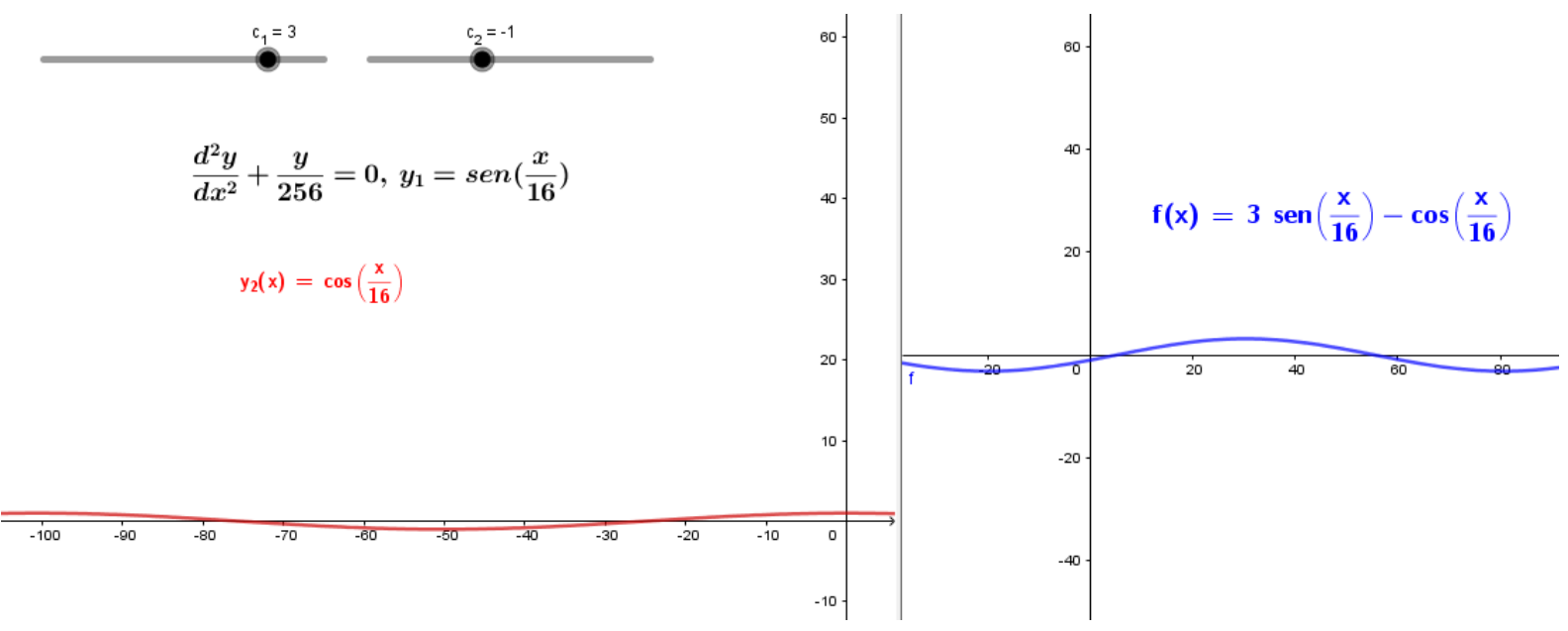

Figure 15

\section{Example 16}

$\mathrm{Be}$

$$
\frac{d^{2} y}{d x^{2}}+\mathrm{y} / 100=0, y_{1}=\operatorname{sen}(x / 10)
$$

In figure 16 the second solution obtained by reduction of order $\cos (\mathrm{x} / 10)$ is given in red and the general solution in blue. Where c_1 and c_2 move between -5 and 5 . 


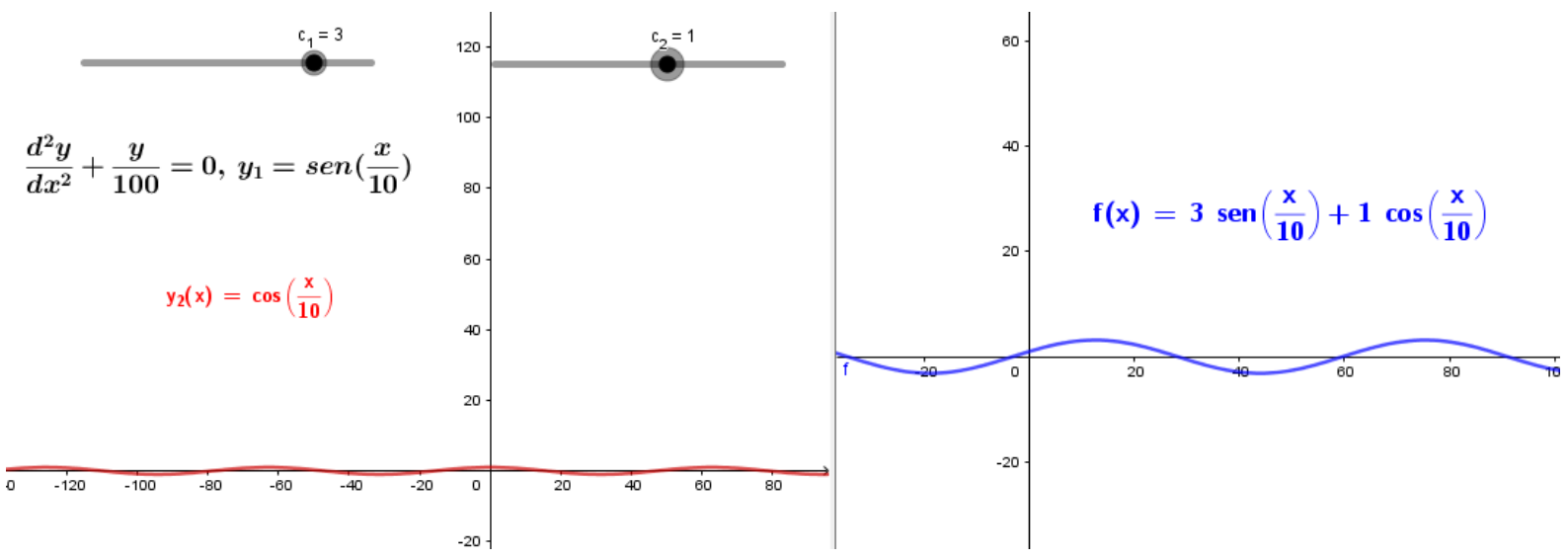

Figure 16

\section{Example 17}

$\mathrm{Be}$

$$
\frac{d^{2} y}{d x^{2}}+\mathrm{y} / 10000=0, y_{1}=\operatorname{sen}(x / 100)
$$

In figure 17 the second solution obtained by reduction of order $\cos (\mathrm{x} / 100)$ is given in red and the general solution in blue. Where c_1 and c_2 move between -5 and 5 .

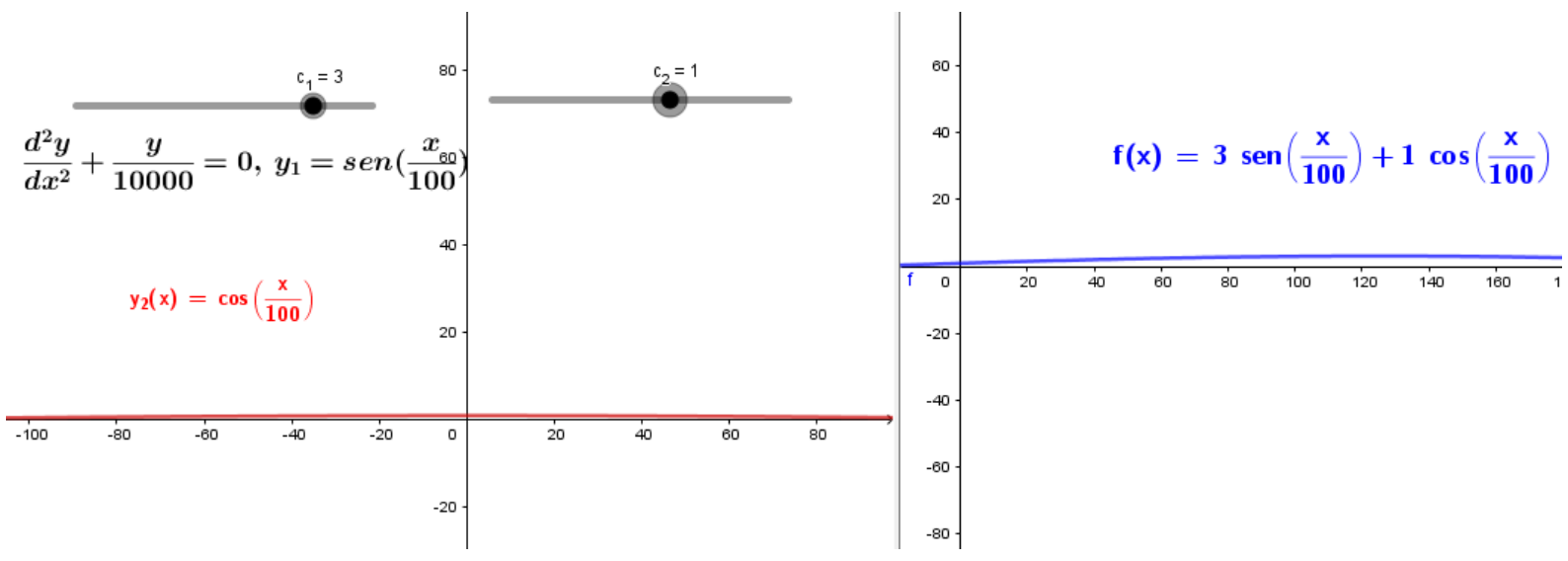

Figure 17

\section{Conclusion}

Our purpose in this work has been to share the importance of using GeoGebra to see the solutions obtained by order reduction in differential equations. 
All these applets designed by GeoGebra correspond to teaching support material, help for students in their learning, these applets that are easy to access can be viewed or downloaded from any computer, tablet or cell phone.

\section{References}

[1] Tom Walsh. Creating interactive physics simulations using the power of GeoGebra. The Physics Teacher 55, 316 (2017); https://doi.org/10.1119/1.4981047

[2] Dan MacIsaac. Example Geogebra physics simulations and animations for physics teaching .The Physics Teacher 55, 384 (2017); doi: 10.1119/1.4999747

[3] Jorge Olivares Funes and Elvis Valero (2018). Animations and interactive creations in linear differential equations of first order: the case of GeoGebra . IOP Conf. Series: Journal of Physics 1141012126. 